027.7 Zeitschrift für Bibliothekskultur / Journal for Library Culture • Digital Humanities und wissenschaftliche Bibliotheken

\title{
Editorial: Digital \\ Humanities und \\ wissenschaftliche \\ Bibliotheken
}

Catrina Langenegger, Iris Lindenmann, Noah Regenass

License: Creative Commons Attribution 4.0 International License (CC-BY 4.0). 
Wissenschaftliche Bibliotheken sind schon seit den Anfängen der Digitalisierung dabei, Forschenden bei der Arbeit mit digitalen Methoden und Daten mit Rat und Tat zur Seite zu stehen. Kenntnisse und Verantwortungen im Bereich Digitalisierung, Forschungsdatenmanagement und Open Publishing sowie der Informationskompetenz (zum Beispiel Datenbankschulungen) sind aus dem Arbeitsalltag nicht mehr wegzudenken. Umso erstaunlicher also, dass der Umgang mit den Digital Humanities (DH) in den Bibliotheken eher zögerlich ist und noch kaum Best-Practice-Modelle existieren. Der Aufbau von Dienstleistungen an Bibliotheken in den DH hängt häufig vom Engagement einzelner Personen und den lokalen Gegebenheiten ab. Dass jedoch Infrastrukturen und Ressourcen für Anfragen rund um DH benötigt werden, ist unumstritten und zeigt sich auch in der Literatur der letzten Jahre (zum Beispiel Hartsell-Gundy, Braunstein, \& Golomb, 2015; Kear \& Joranson, 2018; Wilson, 2020; Chambers, 2021 - ausstehend). Dieses Bedürfnis wurde auch an der Universitätsbibliothek Basel (UB Basel) festgestellt: Es werden vermehrt Aufträge und Fragen zum Thema DH an die UB Basel herangetragen.

Um diese Lücke im Serviceangebot zu füllen, wurde an der UB Basel im Februar 2020 die Arbeitsgruppe Digital Humanities (AG DH) - bestehend aus Fachreferent:innen aus den Fachbereichen Musikwissenschaft, Geschichte, Jüdische Studien, Kunstgeschichte, Ethnologie, Kulturanthropologie und Englische Sprach- und Literaturwissenschaft mit der Absicht gegründet, in einem ersten Schritt vorhandenes Wissen zu DHMethoden und -Tools zu bündeln und in einem Portfolio zu sammeln, sowie in einem zweiten Schritt bedarfsgerechten Wissensaufbau zu betreiben, um bei Anliegen zu DHFragen kompetent Auskunft und Unterstützung bieten zu können.

Einen ersten Einblick in die Thematik erhielt die AG DH durch die Abschlussarbeit von Gero Schreier für den Master of Advanced Studies in Bibliotheks- und

Informationswissenschaft (MAS BIW) an der Universität Zürich (2017-2019)ํ. Die AG nahm Schreiers Untersuchung als Startpunkt ihrer Tätigkeit und hat im Zuge der Diskussionen den ursprünglich geplanten Austausch mit dem Autor zu einer kleinen Tagung erweitert. Ziel war es, sich von Kolleg:innen anderer Universitätsbibliotheken in einem ersten Teil Inputs für die Arbeit und Ausrichtung der AG DH zu holen. Im zweiten Teil wurden die Diskussionen in einem Workshop vertieft. Die Resultate wurden in einer öffentlichen Abschlussrunde den Tagungsteilnehmer:innen und weiteren Interessierten vorgestellt. 
Die Tagung fand am 10. November 2020 hybrid in Basel und über Zoom statt. Für den Vormittag wurden vier Gastredner:innen für Inputreferate und Diskussionen im Plenum eingeladen:

- Gero Schreier (Universitätsbibliothek Bern), Fachreferent und Mitglied des Teams Open Science,

- Andrea Rapp (Universität Darmstadt), Professorin für Germanistik, Mediävistik und Computerphilologie,

- Timo Glaser (Universitätsbibliothek Marburg) Fachreferent; richtete dort ein Digital Learning Lab ein,

- Gerhard Lauer (Universität Basel), Professor für Digital Humanities.

Die vorliegende Ausgabe von 027.7 setzt sich aus drei Beiträgen der Tagung, dem Bericht eines Forschungsprojekts an der Universität Basel, der konkret die Chancen und Herausforderungen der Zusammenarbeit zwischen UB Basel und DH-Projekten hervorhebt, sowie der Zusammenfassung der aus Tagung und Workshop gewonnenen Einsichten der AG zusammen.

Den Anfang macht Andrea Rapp, die in ihrem Beitrag aufzeigt, wie sich Bibliotheken „als Gedächtnisinstitutionen sowie als Labor und Echokammer“ im Rahmen der zunehmend digitalisierten Forschungslandschaft neue Wege eröffnen und dadurch Chancen entstehen, aktiver in Forschungsprojekten beteiligt zu sein.

Timo Glaser setzt sich in seinem Artikel unter anderem für die Betreuung und Beratung auch von individuellen, kleineren Anliegen (im Gegensatz zu grossen „Leuchtturmprojekten“) und die Vermittlung von DH-Kompetenzen bereits auf BA/MAStufe ein. Ebenfalls argumentiert er für einen pragmatischen „learning by doing“Ansatz für den Wissensaufbau, in dem die wissenschaftlichen Bibliothekar:innen Kurse und Workshops anbieten, für die sie selbst Kompetenzen erwerben möchten.

Gerhard Lauer plädiert für eine umfassendere Verbindung von Forschung und Bibliothek und zeigt mit einem Blick zurück in die Geschichte auf, warum Bibliotheken prädestiniert dazu sind, die Forschung bei DH-Projekten zu unterstützen und zu ergänzen. Er weist darauf hin, dass Bibliotheken jetzt die Gelegenheit ergreifen sollten, sich in diesem Bereich zu positionieren.

Als Beispiel aus der Praxis stellt der Beitrag von J. Berenike Herrmann, Giulia Grisot, Susanne Gubser und Elias Kreyenbühl das Projekt „High Mountains“ vor, und wie dafür mittels digitaler Methoden und klassischer philologischer Arbeit ein Korpus 
Deutschschweizer Schriftsteller:innen und deren Werke (Schönliteratur) zwischen 1880-1930 erstellt wurde.

$\mathrm{Zu}$ guter Letzt folgt eine ausformulierte Fassung des Tagungsberichts, den die AG DH im Anschluss an die Veranstaltung der Direktion der UB Basel vorgelegt hat.

Thematisiert werden stichpunktartig die wichtigsten Erkenntnisse und Inputs aus der Tagung und dem Workshop, welche gleichzeitig auch als Anhaltspunkte und Denkanstösse für die weitere Arbeit und die Konzeption des Dienstleistungsportfolios dienen sollen.

Konkrete Antworten auf und Lösungen für die Frage, wie Bibliotheken am besten DHUnterstützung leisten können, wird es keine geben. Dafür umso mehr Motivation, Neugier und Freude, an den Chancen und Herausforderungen der Thematik zu wachsen und Angebote, Wissen und Kompetenzen den Entwicklungen entsprechend aufzubauen. Die AG DH der UB Basel wünscht eine anregende Lektüre.

\section{Footnotes}

1. Das Abstract zu dieser Arbeit ist hier veröffentlicht: https://www.masbiw.uzh.ch/dam/jcr:d5fba131-a2ec-4c24-a645-404ab90cd01d/mas17-

19 abschlussarbeit abstract schreier.pdf $\underline{\underline{\text { df }}} \leftrightharpoons$

\section{Citations}

1. Hartsell-Gundy, A., Braunstein, L., \& Golomb, L. (2015). Digital humanities in the library: challenges and opportunities for subject specialists. Chicago: The Association of College \& Research Libraries. $\_$

2. Kear, R., \& Joranson, K. (Eds.). (2018). Digital Humanities, Libraries, and Partnerships: A Critical Examination of Labor, Networks, and Community. Oxford: Chandos Elsevier. Retrieved from http://d-scholarship.pitt.edu/33878/ 3. Wilson, E. A. (2020). Digital Humanities for Librarians. Lanham: Rowman \& Littlefield. $\doteq$

4. Chambers, S. (Ed.). (2021). Digital humanities : an introduction for librarians. London: Facet. Retrieved from http://hdl.handle.net/1854/LU-8511559 